\title{
Diameter Perfect Lee Codes
}

\author{
Peter Horak, Bader F. AlBdaiwi
}

\begin{abstract}
Lee codes have been intensively studied for more than 40 years. Interest in these codes has been triggered by the Golomb-Welch conjecture on the existence of the perfect errorcorrecting Lee codes. In this paper we deal with the existence and enumeration of diameter perfect Lee codes. As main results we determine all $q$ for which there exists a linear diameter- 4 perfect Lee code of word length $n$ over $Z_{q}$, and prove that for each $n \geq 3$ there are uncountable many diameter- 4 perfect Lee codes of word length $n$ over $Z$. This is in a strict contrast with perfect error-correcting Lee codes of word length $n$ over $Z$ as there is a unique such code for $n=3$, and its is conjectured that this is always the case when $2 n+1$ is a prime. We produce diameter perfect Lee codes by an algebraic construction that is based on a group homomorphism. This will allow us to design an efficient algorithm for their decoding. We hope that this construction will turn out to be useful far beyond the scope of this paper.
\end{abstract}

Index Terms-Diameter codes, error-correcting codes, Lee metric, perfect codes.

\section{INTRODUCTION}

The most common metric in coding theory is the Hamming metric. In this paper we deal with another frequently used metric, so called Lee metric. This metric was introduced in [16] and [27] for transmission of signals. The main motive power for studying codes in Lee metric goes back to the seminal paper of Golomb and Welch [8]. In this paper we will study the existence and will enumerate diameter perfect Lee codes.

First we recall some definitions and notation. As usual, let $Z$ be the set of integers, $Z_{q}$ denote the integers modulo $q$, and let $T^{n}$ be the $n$-fold Cartesian product of a set $T$. Throughout the paper we will use $Z_{q}^{n}$ and $Z^{n}$ for the Abelian (componentwise) additive group on $Z_{q}^{n}$ and $Z^{n}$ as well. It will always be clear from the context whether we have in mind a set or the group on this set. Because of the coding theory context the elements of $Z_{q}^{n}$ and $Z^{n}$ will also be called words. The Lee distance (=the Manhattan distance, the zig-zag distance) $\rho_{L}(v, w)$ of two words $v=\left(v_{1}, v_{2}, \ldots, v_{n}\right), w=\left(w_{1}, \ldots, w_{n}\right)$ is given by $\rho_{L}(v, w)=\sum_{i=1}^{n} \min \left(\left|v_{i}-w_{i}\right|, q-\left|v_{i}-w_{i}\right|\right)$ for $u, v \in \mathbb{Z}_{q}^{n}$, and by $\rho_{L}(v, w)=\sum_{i=1}^{n}\left|v_{i}-w_{i}\right|$ for $v, w \in Z^{n}$. By $S_{n, r}(v)$ we denote the Lee sphere of radius $r$ centered at $v$; that is, $S_{n, r}(v)=\left\{w ; \rho_{L}(v, w) \leq r\right\}$. For $v, w$ with

Peter Horak is with the Interdisciplinary Arts and Sciences, University of Washington, Tacoma, 1900 Commerce St., Tacoma, WA 98402-3100, USA (email: horak@uw.edu).

Bader F. AlBdaiwi is with the Computer Science Department, Kuwait University, P. O. Box 5969, Safat, 13060, Kuwait (email: bader.albdaiwi@ku.edu.kw).
$\rho_{L}(v, w)=1$, the double Lee sphere $D S_{n, r}(v, w)$ of radius $r$ with its center comprising $v, w$ is the set $S_{n, r}(v) \cup S_{n, r}(w)$.

Perfect error-correcting Lee codes can be introduced in several ways. For the purpose of this paper it is convenient to define them through tilings. In order not to have to formulate the same statement/definition twice, once for $Z^{n}$ and then for $Z_{q}^{n}$, the symbol $Z_{*}^{n}$ stands for both of them; that is, by $Z_{*}^{n}$ we mean $Z^{n}$ or $Z_{q}^{n}$. We set $O=(0, \ldots, 0)$ and $e_{i}=(0, \ldots, 0,1,0, \ldots, 0)$, where the $i$-th coordinate equals 1 .

Let $V$ be a subset of $Z_{*}^{n}$. By a copy of $V$ we mean an image of $V$ under a linear distance preserving bijection on $Z_{*}^{n}$. Translations of $V$ form a special family of copies of $V$. We recall that by a translation of $V$ we understand the set $V+x=\{w ; w=x+v, v \in V\}$ where $x \in Z_{*}^{n}$. As the sphere $S_{n, r}$ is symmetric, each copy of the sphere is its translation. Each copy of the double-sphere $D S_{n, r}$ can be obtained by a translation of the double-sphere $D S_{n, r}\left(O, e_{i}\right)$ for some $i, 1 \leq$ $i \leq n$. A collection $\mathcal{T}=\left\{V_{i} ; i \in I\right\}$ of copies of $V$ constitutes a tiling of $Z_{*}^{n}$ by $V$ if $\bigcup_{i \in I} V_{i}=Z_{*}^{n}$ and $V_{i} \cap V_{j}=\emptyset$ for all $i \neq j$. A tiling $\mathcal{T}$ of $Z_{*}^{n}$ by $V$ that consists of translations of $V$ can be described as $\mathcal{T}=\{V+l ; l \in \mathcal{L}\}$ where $\mathcal{L} \subset Z_{*}^{n}$. A tiling $\mathcal{T}$ by translations of $V$ is called periodic if $\mathcal{L}$ is periodic, and $\mathcal{T}$ is called a lattice tiling if $\mathcal{L}$ is a lattice (=a subgroup) of $Z_{*}^{n}$.

Let $V=S_{n, r}$ in $Z_{*}^{n}$ such that, if $Z_{*}^{n}=Z_{q}^{n}$ then $q \geq 2 r+1$. A set $\mathcal{L} \subset Z_{*}^{n}$ is called a perfect $r$-error-correcting Lee code if $\mathcal{T}=\{V+l ; l \in \mathcal{L}\}$ is a tiling of $Z_{*}^{n}$. If $\mathcal{L}$ is a lattice then $\mathcal{L}$ is called a linear code. For $Z_{*}^{n}=Z^{n}$ the perfect $r$-errorcorrecting Lee code is denoted by $P L(n, r)$ while for $Z_{*}^{n}=$ $Z_{q}^{n}$ it is denoted by $P L(n, r, q)$. The long-standing GolombWelch conjecture deals with the existence of $P L(n, r)$ codes. Although there is a vast literature on the subject the conjecture is still far from being solved.

In this paper we focus on diameter- $d$ perfect Lee codes, which constitute a generalization of perfect error-correcting Lee codes. Ahlswede et al., see [2], introduced diameter perfect codes for distance regular graphs. Let $(M, \rho)$ be a metric space. Then a set $C \subset M$ is a diameter- $d$ code if $\rho(u, v) \geq d$ for any $u, v \in C$, and a set $A \subset M$ is an anticode of diameter $d$ if $\rho(u, v) \leq d$ for all $u, v \in A$. Each graph $G$ can be seen as a metric space. It is proved in [2] that if $G$ is a distance regular graph, $C$ is a diameter- $d$ code in $G$, and $A$ is an anticode of diameter $d-1$ of the maximum size in $G$ then $|C||A| \leq|G|$; that is, the sphere packing bound applies to distance regular graphs as well. A diameter- $d$ code that attains the bound in the above inequality is called diameter- $d$ perfect code. This definition can be used for example in Hamming 
scheme as the related graph is distance regular. Etzion [7] showed that the sphere packing bound applies to $Z_{q}^{n}$ endowed with Lee metric as well although the related graph is not distance regular; analogically to [2] he defined the diameter- $d$ perfect Lee code as a diameter- $d$ code that attains the equality in the sphere packing bound. Clearly, a definition through the sphere packing bound cannot be extended to an infinite space. To deal with the case of $Z^{n}$ Etzion proved that if $\mathcal{L}$ is a lattice in $Z^{n}$ with the minimum distance $d$ and a volume $|V(\mathcal{L})|$ then $|A| \leq|V(\mathcal{L})|$ for any anticode of diameter $d-1$. Then he called $\mathcal{L}$ to be the diameter perfect code if $\mathcal{L}$ attains the equality in the bound. As far as we know there is no formal definition of the diameter perfect Lee code in $Z^{n}$ in case when $\mathcal{L}$ is not a lattice.

Now we provide a unified definition of a diameter- $d$ perfect Lee code for both spaces $Z_{q}^{n}$ and $Z^{n}$ regardless whether the code is a linear one. Let $\mathcal{S}=\left\{S_{i} ; i \in I\right\}$ be a family of subsets of an underlying set $M$. Then a set $T \subset M$ is called a transversal of $\mathcal{S}$ if there is a bijection $f: I \rightarrow T$ so that $f(i) \in S_{i}$. Equivalently, $T$ is a transversal of $\mathcal{S}$ if $\left|T \cap S_{i}\right|=1$ for each $i \in I$, and $T \cap S_{i} \neq T \cap S_{j}$ for all $i \neq j \in I$.

Definition 1: Let $\mathcal{L} \subset Z_{*}^{n}$. Then $\mathcal{L}$ is a diameter- $d$ perfect Lee code in $Z_{*}^{n}$ if $\mathcal{L}$ is a diameter- $d$ code, and there is a tiling $\mathcal{T}=\left\{W_{i} ; i \in I\right\}$ of $Z_{*}^{n}$ by the anticode of diameter $d-1$ of maximum size such that $\mathcal{L}$ is a transversal of $\mathcal{T}$. The diameter$d$ perfect Lee code in $Z^{n}$ will be denoted by $D P L(n, d)$, and in $Z_{q}^{n}$ by $D P L(n, d, q)$.

In other words, a diameter- $d$ code $\mathcal{L}$ is perfect if there is a tiling $\mathcal{T}$ by anticodes of diameter $d-1$ of the maximum size so that each tile in $\mathcal{T}$ contains exactly one codeword of $\mathcal{L}$. It is not difficult to see that for $Z_{q}^{n}$ and for lattice case in $Z^{n}$ our definition of diameter perfect Lee codes is equivalent to the definition due to Etzion.

Any error-correcting perfect Lee code is also a diameter perfect Lee code. Indeed, it is easy to see that, for $d$ even, the anticode of diameter $d$ of the maximum size is the Lee sphere $S_{n, r}$ with $r=\frac{d}{2}$. Thus, for $d$ odd, $D P L(n, d)$ and $D P L(n, d, q)$ codes are $P L(n, r)$ and $P L(n, r, q)$ codes where $r=\frac{d-1}{2}$, respectively. It was proved in [3] that, for $d$ odd, the anticode of diameter $d$ of maximum size is the double-sphere $D S_{n, r}$ with $r=\frac{d-1}{2}$.

We point out an advantage of our definition. Suppose that we need to show the existence of a diameter- $d$ perfect Lee code in $Z_{*}^{n}$ where $d$ is even. Then all we need to do is to prove that there is a tiling $\mathcal{T}=\left\{W_{i} ; i \in I\right\}$ of $Z_{*}^{n}$ by the double-sphere $D S_{n, r}$ of radius $r=\frac{d-2}{2}$. Indeed, choose a fixed tile $W_{i_{0}}$ in $\mathcal{T}$. Let $x$ be one of the two words forming the center of $W_{i_{0}}$. We will prove that the set $N=\left\{y ; y\right.$ belongs to the center of $W_{i}$ and $\rho_{L}(x, y)$ is even, $\left.i \in I\right\}$ is a distance- $d$ perfect Lee code. Clearly, $N$ is well defined as the center of $W_{i}$ consists of two words at distance 1 ; that is, one of them at even and the other at odd distance from $x . N$ is a transversal of $\mathcal{T}$, thus we need only to prove that $\rho_{L}(x, y) \geq d$ for any $x, y \in N$. Clearly, the distance of any two words in $N$ is even. This is obvious to see for $Z_{*}^{n}=Z^{n}$. It is not true in general for $Z_{*}^{n}=Z_{q}^{n}$, it is valid only in the case when $q$ is even. However, as the size of $D S_{n, r}$ is even (see [7]) $\left|Z_{q}^{n}\right|=q^{n}$ has to be even as well, which in turn implies $q$ is even. So for the distance of any two words in $N$ we have $\rho_{L}(x, y) \geq r+r+1$; and since $\rho_{L}(x, y)$ is even, we get $\rho_{L}(x, y) \geq 2 r+2=d$. We have proved:

Theorem 2: If there is a tiling of $Z_{*}^{n}$ by double-spheres $D S_{n, r}$ then there is a distance- $d$ perfect Lee code in $Z_{*}^{n}$ with $d=2 r+2$. Moreover, if $\{W+l ; l \in \mathcal{L}\}$ is a tiling of $Z_{*}^{n}$ by translations of $D S_{n, r}$ such that the weight $|l|=\rho_{L}(l, O)$ of $l$ is even for all $l \in \mathcal{L}$ then $\mathcal{L}$ is a diameter- $d$ perfect Lee code.

The main goal of this paper is to study the existence and to enumerate diameter perfect Lee codes. It turns out that some results on diameter perfect Lee codes and on perfect errorcorrecting Lee codes are similar but in some cases the results on the two types of codes are very much different. E.g., it was proved in [15] that there is only one, up to isomorphism, tiling of $Z^{3}$ by Lee spheres $S_{3,1}$ but we show in this paper that there are uncountable many tilings of $Z^{3}$ by double Lee sphere $D S_{3,1}$. The rest of the paper is organized as follows. Golomb-Welch conjecture claims that there are no $P L(n, r)$ codes for $n \geq 3$, and $r>1$. The current state of this conjecture as well as its extension to $D P L(n, d)$ codes and a further extension to perfect dominating sets is provided in Section 2. We defined perfect Lee codes by means of tilings. In [23] S. Stein introduced an algebraic construction of lattice tilings based on group homomorphisms. He used this construction for tilings by crosses. This construction, and its variations occur in many papers, see e.g. [4], [10], [11], [15], [18], [22], [24], [25], [26]. In Section 3 a generalization of Stein's construction is given. Let $V$ be a subset of $Z^{n}$. We state a necessary and sufficient condition for the existence of a lattice tiling of $Z^{n}$ by $V$ in terms of a group homomorphism. We guess that this construction will turn useful outside the scope of this paper.

The main results of this paper are stated in Section 4. It is believed, see Section 2, that, with only one exception of $D P L(3,6)$ code, there are no $D P L(n, d)$ codes, and also no $D P L(n, d, q)$ codes for $n \geq 3$, and $d>4$. All values of $q$ for which there exists a linear $P L(n, 1, q)$ code (=a linear $D P L(n, 3, q)$ code) were determined in [1]. We prove an analogous result for linear $\operatorname{DPL}(n, 4, q)$ codes. As to the enumeration of all $D P L(n, 4)$ codes, Etzion [7] proved that there are uncountable many of them if $n$ is a power of 2 . We show that this is also the case for the other values of $n \geq 3$. The situation with $P L(n, 1)$ codes is very much different. It was proved in [15] that there are uncountable many $P L(n, 1)$ codes if $2 n+1$ is not a prime. However, on the other hand it was showed there that there is only one, up to isomorphism, $P L(3,1)$ code, and the authors conjectured that the same is true for each $n$ where $2 n+1$ is a prime. We note that the existence of uncountable many $D P L(n, 4)$ codes implies that there are uncountable many non-periodic $D P L(n, 4)$ codes as the total number of periodic $D P L(n, 4)$ codes is at most $\aleph_{0}$. Non-periodic $\operatorname{DPL}(n, 4)$ codes were constructed by means 
of non-regular lattice tilings (i.e. tilings that are not a face-toface tilings) of $R^{n}$ by a cluster of cubes centered at words of a double-sphere $D S_{n, 1}$. This is another example of a well know situation when to solve a problem we need to generalize it first. We note that results in Section 4 answer in the affirmative questions (4), (5), and (8) raised by Etzion in [7]. Question (6) from [7] was answered in [15]. Finally, in Section 5 an efficient decoding algorithm for linear perfect Lee codes, both diameter and error-correcting, is designed. Thanks to the representation of these codes by tilings constructed via a group homomorphism, the computation complexity of this algorithm is $\Theta(n)$ for $D P L(n, d), d=3,4$, codes, and its complexity is $O(\log d)$ for $D P L(2, d)$ codes. We recall that $D P L(n, d)$ codes are believed not to exists for $n \geq 3$ and $d>4$.

\section{GOLOMB-WELCH CONJECTURE AND ITS EXTENSIONS}

In this section we provide a short account of the current state of Golomb-Welch conjecture and some of its extensions. In [8] it is conjectured that:

Conjecture 3: There is no $P L(n, r)$ code for $n \geq 3$ and $r>1$.

If true then the conjecture is best possible as the existence of $P L(n, r)$ codes for $n=2$ and all $r \geq 1$, and $n \geq 3$ and $r=1$ was showed by several authors, see e.g. [8]. It is also proved in [8] that for each $n \geq 3$ there is $r_{n}, r_{n}$ not specified, so that there is no $P L(n, r)$ code with $r \geq r_{n}$. Although there are plenty of results in the literature on this conjecture the non-existence of $P L(n, r)$ codes has been proved only in few cases. First, Gravier et al. [9] settled the Golomb-Welch conjecture for $n=3$ and all $r>1$. Later Špacapan [21], whose proof is computer aided, showed the non-existence of a $P L(n, r)$ code for $n=4$ and all $r>1$. Horak [13] provided an algebraic proof that there is no $P L(n, r)$ code for $3 \leq n \leq$ 5 and all $r>1$.The only other value of parameters for which the Golomb-Welch conjecture is known to be true is $n=6$ and $r=2$, see [12].

The non-existence of $P L(n, r, q)$ codes for some pairs $(n, r)$ and specific values of $q$ depending on $(n, r)$ was stated in several papers, see e.g. [5] for a comprehensive account. The best result of this type is due to Post [19] who showed that there is no $P L(n, r, q)$ code for any $q \geq 2 r+1$ (= code with a large alphabet), and $3 \leq n \leq 5, r \geq n-2$, and for $n \geq 6$, and $r \geq \frac{\sqrt{2}}{2} n-\frac{1}{4}(3 \sqrt{2}-2)$. Post's result can also be viewed as follows: For the given pairs of $(n, r)$ there is no periodic $P L(n, r)$ code. In [12] and [14] the first author of this paper quoted incorrectly Post's result as he omitted the word periodic.

As mentioned in the Introduction, $D P L(n, d)$ codes are a generalization of $P L(n, r)$ codes as, for $d$ odd, a $D P L(n, d)$ code is a $P L\left(n, \frac{d-1}{2}\right)$ code as well. Therefore the conjecture stated by Etzion in [7] is an extension of the Golomb-Welch conjecture.
Conjecture 4: [7] There is no $\operatorname{DPL}(n, d)$ code for $n \geq 3$ and $d>4$ with the exception of the pair $(n, r)=(3,6)$.

Etzion's conjecture, if true, is also best possible. The existence of $D P L(2, d)$ codes, for $d$ odd was stated in [8] in terms of $P L(n, r)$ codes. The existence of $\operatorname{DPL}(n, d)$ codes for $n=2$ and all even $d \geq 4$, and all $n \geq 3$ and $d=4$ was established in [7] and [4], while the existence of a $\operatorname{DPL}(3,6)$ code follows from a Minkowski tiling [17].

To be able to present an extension of Etzion's conjecture we state a generalization of diameter perfect Lee codes introduced in [4]. Let $G=(V, E)$ be a graph, and $t$ be a natural number. A set $S \subset V$ is called a t-perfect distance-dominating set in $G$, or a $t$-PDDS in $G$, if, for each $v \in V$, there is a unique component $C_{v}$ of $[S]$ so that $d\left(v, C_{v}\right) \leq t$, and there is in $C_{v}$ a unique vertex $w$ with $d(v, w)=d\left(v, C_{v}\right)$. We recall that the distance $d(v, C)$ of a vertex $v \in V$ to $C \subset V$ is given by $d(v, C)=\min \{d(v, w) ; w \in C\}$ and that $[S]$ stands for the subgraph of $G$ induced by $S$. To facilitate our discussion, a $t$-PDDS in $G$ all whose components are isomorphic to a graph $H$ will be denoted by $t$-PDDS $[H]$.

Let $\Lambda_{n}$ be the integer grid, that is, $\Lambda_{n}$ has $Z^{n}$ as its vertex set with two vertices being adjacent if their distance equals 1. Let $P_{k}$ stand for a path on $k$ vertices. Thus $P_{1}$ is a single vertex and $P_{2}$ is a pair of adjacent vertices. It is not difficult to see that an $r$-PDDS $\left[P_{1}\right]$ in $\Lambda_{n}$ constitutes a $P L(n, r)$ code while an $r$-PDDS $\left[P_{2}\right]$ in $\Lambda_{n}$ is a $\operatorname{DPL}(n, 2 r+2)$ code. In [4] it is conjectured that:

Conjecture 5: Let $k \geq 1$. Then there is no $r$-PDDS $\left[P_{k}\right]$ in $\Lambda_{n}$ for $n \geq 3$ and $r>1$ with the exception of 2-PDDS $\left[P_{2}\right]$ in $\Lambda_{3}$.

As with the Golomb-Welch and the Etzion conjecture, if true, then Conjecture 5 is best possible. The existence of $t$-PDDS $\left[P_{k}\right]$ in $\Lambda_{n}$ for $n=2, k \geq 1, t \geq 1$, and for $n \geq 3, k \geq 1, t=1$ has been proved in [4], while 2-PDDS $\left[P_{2}\right]$ in $\Lambda_{3}$ is the $\operatorname{DPL}(3,6)$ code mentioned above.

At the end of this section we note that in [4] there are other results and a conjecture on the existence of $t$-PDDS $[H]$ sets in $\Lambda_{n}$ outside the scope of diameter perfect Lee codes.

\section{LATtice Tilings of $Z^{n}$.}

For the purpose of this paper it was convenient to define perfect Lee codes by means of tilings. The main reason was that with this definition in hand we will be able to prove the existence of certain codes using a construction of lattice tilings through group homomorphism. We believe that Stein in [23] was the first one to use graph homomorphisms to produce a lattice tiling, in this case a tiling by crosses. Variations of Stein's construction can be found in many papers, see [4], [10], [11], [15], [18], [22], [24], [25], [26], where the authors use Stein's approach for lattice tilings by different types of crosses and spheres. In this section a generalization of Stein's construction is given. We first describe this construction and 


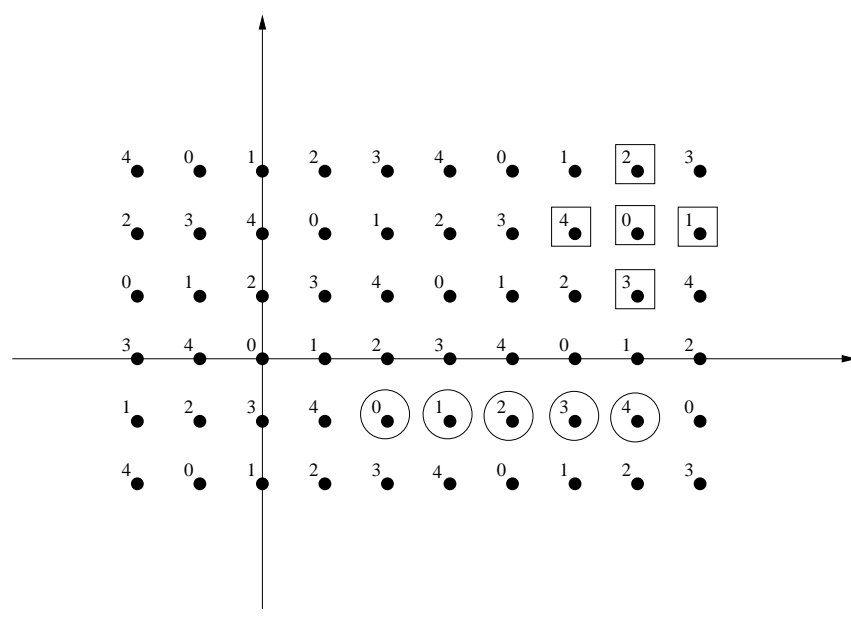

Fig. 1: Set $V$

then we provide a necessary and sufficient condition that allows one to verify whether there is a lattice tiling of $Z^{n}$ by a given subset.

Let $\mathcal{L}$ be a lattice (=subgroup) of $Z^{n}$. Consider the factor group $G=Z^{n} / \mathcal{L}$. Two elements $u, v \in Z^{n}$ belong to the same coset of $Z^{n} / \mathcal{L}$ (that is, they are two different representations of the same element $g \in G$ ) if $u-v \in \mathcal{L}$. Choose one element $v_{g} \in Z^{n}$ from each coset of $Z^{n} / \mathcal{L}$ (=for each element of the factor group $G$ ) and put $V:=\left\{v_{g} ; v \in G\right\}$. Then the collection $\mathcal{T}=\{V+l ; l \in \mathcal{L}\}$ forms a partition of $Z^{n}$ by translations of $V$. As $\mathcal{L}$ is a lattice $\mathcal{T}$ constitutes a lattice tiling of $Z^{n}$ by $V$. A different choice of the elements $v_{g}$, although considering the same lattice $\mathcal{L}$, provides tilings of $Z^{n}$ by different sets $V$. Consider a homomorphism $\phi: Z^{2} \rightarrow Z_{5}$, where $\phi\left(e_{1}\right)=1$, and $\phi\left(e_{2}\right)=2$. In Fig. 1 it is shown that we can choose $V$ to be a copy of the set $\left\{t e_{i} ; 0 \leq t \leq 4\right\}$ as well as $V$ to be a copy of the Lee sphere $S_{2,1}$. However, we are interested in the "inverse" process. Given a set $V$, find a lattice tiling $\mathcal{T}=\{V+l ; l \in \mathcal{L}\}$ of $Z^{n}$ by $V$. To be able to apply the above construction we need to find a lattice $\mathcal{L}$ so that it would be possible to choose elements $v_{g}$ from individual cosets of $Z^{n} / \mathcal{L}$ forming a copy of the set $V$. However, to find a lattice with the required properties might be a painstaking process. It is nearly impossible to do it by trying all options as usually there are infinitely many of them. To remedy the problem the following theorem provides a way how to construct the required tiling $\mathcal{T}$ without knowing the lattice $\mathcal{L}$ explicitly. It turns out that all what is needed is to find a suitable Abelian group $G$ of order $V$ and a homomorphism $\phi: Z^{n} \rightarrow G$ satisfying a condition that can be easily verified.

Theorem 6: Let $V$ be a subset of $Z^{n}$. Then there is a lattice tiling $\mathcal{T}$ of $Z^{n}$ by $V$ if and only if there is an Abelian group $G$ of order $|V|$ and a homomorphism $\phi: Z^{n} \rightarrow G$ so that the restriction of $\phi$ to $V$ is a bijection.

Proof: Let $G$ be an Abelian group and let $\phi: Z^{n} \rightarrow G$ be a homomorphism so that the restriction of $\phi$ to $V$ is a bijection. It is well known that $\operatorname{ker}(\phi)=\mathcal{L}$ is a subgroup of $Z^{n}$ and $Z^{n} / \mathcal{L}=G$. As mentioned above, two elements $x, y \in Z^{n}$ belong to the same coset of the factor group $Z^{n} / \mathcal{L}$ iff $x-y \in \mathcal{L}$. To show that $\mathcal{T}=\{V+l ; l \in \mathcal{L}\}$ is a tiling of $Z^{n}$ by $V$ we need to prove that (i) $\bigcup_{l \in \mathcal{L}} V+l=Z^{n}$, (ii) $V+l \cap V+l^{\prime}=\emptyset$ for any $l, l^{\prime} \in \mathcal{L}, l \neq l^{\prime}$. Assume that there is $x \in Z^{n}$ so that $x \notin \bigcup_{l \in \mathcal{L}} V+l$. Let $\phi(x)=g$. Since the restriction of $\phi$ to $V$ is a bijection, there is $y \in V$ so that $\phi(y)=\dot{g}$; that is, $x$ and $y$ belong to the same coset of $Z^{n} / \mathcal{L}$. Thus, $x-y=l \in \mathcal{L}$, which in turn implies, as $y \in V$, that $x \in V+l ; l \in \mathcal{L}$, a contradiction. The proof of (i) is complete. Assume now that there are $l, l^{\prime} \in \mathcal{L}, l \neq l^{\prime}$, such that there is $x$ in $Z^{n}$ with $x \in V+l \cap V+l^{\prime}$. Hence $x$ can be written as $x=y+l$ and also as $x=y^{\prime}+l^{\prime}$ for some $y \neq y^{\prime} \in V$. We have $y+l=y^{\prime}+l^{\prime}$, that is $y-y^{\prime}=l^{\prime}-l \in \mathcal{L}$. However, $y-y^{\prime} \in \mathcal{L}$ implies $\phi\left(y-y^{\prime}\right)=0$, that is $\phi(y)=\phi\left(y^{\prime}\right)$, a contradiction as $\phi$ is a bijection on $V$.

To prove the necessary part of the condition, assume that $\mathcal{T}=\{V+l ; l \in \mathcal{L}\}$ is a lattice tiling of $Z^{n}$ by $V$; that is, $\mathcal{L}$ is a subgroup of $Z^{n}$. Consider the factor group $G=Z^{n} / \mathcal{L}$ and the mapping $\phi: Z^{n} \rightarrow G$ given by $\phi(x)=g=[x]$, where $[x]$ is the coset of the factor group $Z^{n} / \mathcal{L}$ containing $x$. It is well known that $\phi$ is a homomorphism. To finish the proof we need to show that the restriction of $\phi$ to $V$ is a bijection. Assume that there are $x, y \in V$ with $\phi(x)=\phi(y)$. Then both $x, y$ belong to the same coset of $Z^{n} / \mathcal{L}$, hence $x-y=l \in \mathcal{L}, l \neq 0$. This implies that $x \in V+l=V+(x-y)$ as $V$ contains $y$. We arrived at a contradiction that $\mathcal{T}=\{V+l ; l \in \mathcal{L}\}$ is a tiling because $x \in V+O \cap V+l$, where $l \neq O$. The proof is complete.

We recall that a set $\mathcal{S} \subset Z_{*}^{n}$ is $p$-periodic, $p>0$, if $s \in \mathcal{S}$ iff $s+p e_{i} \in \mathcal{S}$ for all $i=1, \ldots, n$, and $p$ is the smallest number with the property.

Corollary 7: Let $\mathcal{T}$ be a lattice tiling of $Z^{n}$ by $V$ given by the homomorphism $\phi: Z^{n} \rightarrow G$. Then $\mathcal{T}$ is $p$-periodic, where $p=$ l.c.m. $\left\{\operatorname{ord}\left(\phi\left(e_{1}\right)\right), \ldots, \operatorname{ord}\left(\phi\left(e_{n}\right)\right)\right\}$, where $\operatorname{ord}(g)$ stands for the order of the element $g$ in the group $G$.

Proof: Let $\mathcal{T}=\{V+l ; l \in \mathcal{L}\}$, where $\mathcal{L}=\operatorname{ker}(\phi)$. It is well known that each lattice is periodic. To find the smallest number $p$ for which $\mathcal{L}$ is periodic is equivalent to find the smallest $p$ so that $p e_{i} \in \mathcal{L}$ for all $i=1, \ldots, n$. The smallest number $p_{i}>0$ for which $p_{i} e_{i} \in \mathcal{L}$ equals the smallest number for which $\phi\left(p_{i} e_{i}\right)=\phi\left(e_{i}\right)^{p_{i}}=0$ (in the group $G$ ); i.e., equals $\operatorname{ord}\left(\phi\left(e_{i}\right)\right)$. Therefore, the smallest number $p$ so that $p e_{i} \in \mathcal{L}$ for all $i=1, \ldots, n$, equals l.c.m. $\left\{\operatorname{ord}\left(\phi\left(e_{1}\right)\right), \ldots, \operatorname{ord}\left(\phi\left(e_{n}\right)\right)\right\}$.

Now we list some properties of a homomorphism $\phi: Z^{n} \rightarrow G$ in the above theorem that are useful to verify the existence of a required tiling. The first of them claims that $\phi$ is fully determined by images of $e_{i}, i=1, \ldots, n$, under $\phi$.

Condition 8: If a mapping $\phi: Z^{n} \rightarrow(G, \circ)$ is a homomorphism then, for any $a=\left(a_{1}, \ldots, a_{n}\right) \in Z^{n}$, it is $\phi\left(\left(a_{1}, \ldots, a_{n}\right)=\phi\left(e_{1}\right)^{a_{1}} \circ \ldots \circ \phi\left(e_{n}\right)^{a_{n}}\right.$. 


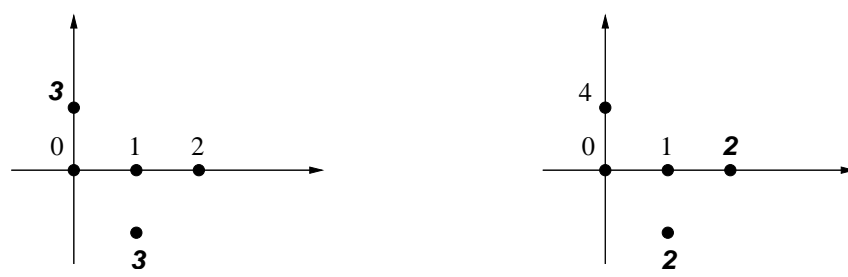

Fig. 2: The restriction of $\phi$ to $V$

Proof: The proof follows from the fact that $\phi(u+v)=$ $\phi(u) \circ \phi(v)$ for any homomorphism $\phi$ and that $a=\left(a_{1}, \ldots, a_{n}\right)$ can be written as $a=a_{1} e_{1}+\ldots+a_{n} e_{n}$.

The following property is clearly true for all tilings and not only for lattice ones. It allows to cut down significantly on the number of cases if one wishes to prove the non-existence of a certain tiling. The proof is obvious and therefore left to the reader.

Condition 9: If $\mathcal{T}=\{V+l ; l \in \mathcal{L}\}$ is a tiling of $Z^{n}$ by $V$ then there is a tiling of $Z^{n}$ of the form $\{W+l ; l \in \mathcal{L}\}$ for any translation $W$ of $V$.

Based on Conditions 8 and 9 the following procedure enables one to find a lattice tiling of $Z^{n}$ by a set $V$ or to prove the non-existence of such tiling.

(i) Take arbitrary copy $W$ of $V$. It is convenient to choose $W$ so that $O \in W$, and $e_{i} \in W$ for as many $i=1, \ldots, n$ as possible.

(ii) Choose an Abelian group $G$ of order $|V|$.

(iii) Choose a homomorphism $\phi: Z^{n} \rightarrow G$; i.e., choose $n$ elements of $G$ as $\phi\left(e_{i}\right)$. Check whether the restriction of $\phi$ to $W$ is a bijection.

As there are finitely many Abelian groups of a fixed finite order the above procedure is finite. The computational complexity depends on the size of $V$, and on the number of Abelian groups of the given order.

We illustrate the procedure by two simple examples. In Fig. 1, it is shown how to choose $\phi\left(e_{i}\right)$ to get a tiling of $Z^{2}$ into Lee spheres $S_{2,1}$. Consider now the set $V=\left\{O, e_{1}, 2 e_{1}, e_{2}, e_{1}-\right.$ $\left.e_{2}\right\}$. We will show that there is no lattice tiling of $Z^{n}$ by $\dot{V}$. Suppose that there is a lattice tiling by $V$. Then there is a homomorphism $\phi: Z^{n} \rightarrow G$ with required properties. Clearly $G=Z_{5}$ as there is only one Abelian group of order 5 . To cut down on the number of cases we note that wlog we can set $\phi\left(e_{1}\right)=1$. Indeed, all elements of $Z_{5}$ are its generators, therefore there exists an automorphism $\psi$ on $Z_{5}$ with $\psi(1)=a$ for any $a \neq 0 \in Z_{5}$. We are left with two choices, namely 3 and 4 , for $\phi\left(e_{2}\right)$. However, in both case the restriction of $\phi$ to $V$ is not a bijection, see Fig. 2. Thus, there is no lattice tiling of $Z^{n}$ by $V$.

To be able to enumerate all - not only lattice - diameter4 perfect Lee codes in $Z^{n}$, we will describe a construction of non-lattice tilings of $Z^{n}$. It turns out that a variation of Theorem 6 might be even used as a starting point to produce such tilings of $Z^{n}$. To do so we need to consider a generalization of the problem to a super-lattice of $Z^{n}$. This is a

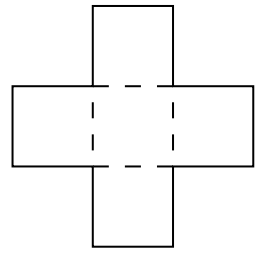

(a) $n=2, r=1$

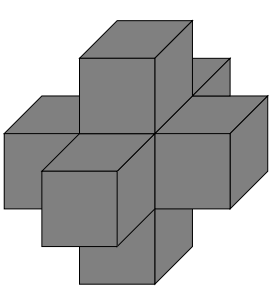

(c) $n=3, r=1$

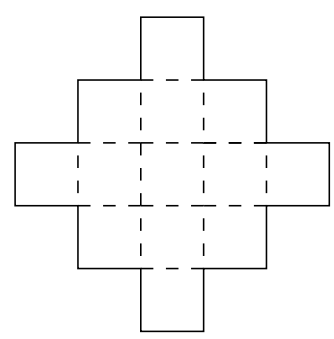

(b) $n=2, r=2$

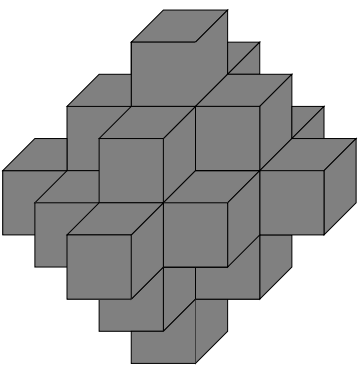

(d) $n=3, r=2$
Fig. 3: Cubistic cross-polytopes

quite common situation in mathematics when a problem has to be generalized before we are able to find its solution. To have a geometric interpretation of this generalization, instead of tiling $Z^{n}$ by a finite set of words $V$, we will consider tilings of Euclidean $n$-space $\mathbb{R}^{n}$ by clusters of unit cubes. In this setting a tiling of $Z^{n}$ by $V$ generates a tiling of $\mathbb{R}^{n}$ by a cluster $C$ of unit cubes so that each word of $V$ is a center of a unit cube in $C$. E.g., a tiling of $Z^{n}$ by a sphere $S_{n, r}$ generates a tiling of $\mathbb{R}^{n}$ by cubistic cross-polytopes, see Fig. 3 for $n=2,3$, and $r=1,2$. We note that the cubistic cross polytope with $r=1$ is also called $n$-cross. Clearly, a tiling $\mathcal{T}$ of $\mathbb{R}^{n}$ by $C$ generated by a tiling of $Z^{n}$ has the following property: Let $T, T^{\prime}$ be two tiles in $\mathcal{T}$ with cubes $C \in T$ and $C^{\prime} \in T^{\prime}$. If $C$ and $C^{\prime}$ have a non-empty $(n-1)$-dimensional intersection then $C$ and $C^{\prime}$ are neighbors; this type of tiling is also called regular or a faceto-face tiling. We recall that two cubes $C, C^{\prime}$ are neighbors if their centers $c=\left(c_{1}, \ldots, c_{n}\right), c^{\prime}=\left(c_{1}^{\prime}, \ldots, c_{n}^{\prime}\right)$ have the following property: there is an index $i$ with $\left|c_{i}-c_{i}^{\prime}\right|=1$ and $c_{j}=c_{j}^{\prime}$ for $i \neq j$. Thus, a non-regular tiling $T=\left\{T_{i}, i \in I\right\}$ of $\mathbb{R}^{n}$ by a cluster of cubes $C$ contains two cubes $C \in T$ and $C^{\prime} \in T^{\prime}, T \neq T^{\prime}$ so that $C$ and $C^{\prime}$ have a non-empty $(n-1)$-dimensional intersection but they are not neighbors. See Fig. 4 for an example of two unit cubes with a nonempty $(n-1)$ dimensional intersection that are not neighbors for $n=2,3$. It turns out that non-regular lattice tilings of $\mathbb{R}^{n}$ by a suitable cluster of cubes will allow us to construct non-lattice, and even non-periodic tilings of $Z^{n}$ by spheres $S_{n, 1}$ and double-spheres $D S_{n, 1}$. We will use the following straightforward generalization of Theorem 6

Theorem 10: Let $V$ be a subset of a lattice $\mathcal{M} \subset \mathbb{R}^{n}$. Then there exists a lattice tiling $\mathcal{T}$ of $\mathcal{M}$ by $V$ if and only if there is an Abelian group $G$ of order $|V|$ and a homomorphism $\phi: \mathcal{M} \rightarrow G$ so that the restriction of $\phi$ to $V$ is a bijection. 


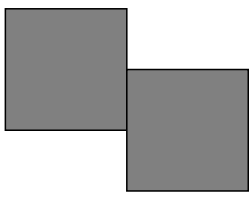

(a) $n=2$

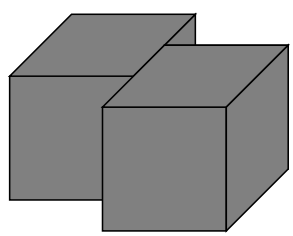

(b) $n=3$
Fig. 4: Cubes having non-empty $(n-1)$ dimensional intersection that are not neighbors

Theorem 10 can be proved by mimicking the proof of Theorem 6 To construct a non-regular tiling we will choose $\mathcal{M}$ to be the lattice generated by vectors $\frac{1}{2} e_{1}, e_{2}, \ldots, e_{n}$. Then a tiling $\mathcal{T}$ of $\mathbb{R}^{n}$ by a cluster of cubes with their centers in $\mathcal{M}$ will be non-regular if and only if there are in $\mathcal{T}$ a cube $C$ with its center at an integral point and a cube $C^{\prime}$ with its center at a point whose first coordinate is of form $m+0.5, m \in Z$. Theorem 11 provides a sufficient condition for the existence of such non-regular tiling of $\mathbb{R}^{n}$ by a cluster of cubes.

Theorem 11: Let $C$ be a cluster of cubes centered at $V \subset$ $Z^{n}$, and $\mathcal{M}$ be a lattice generated by vectors $\frac{1}{2} e_{1}, e_{2}, \ldots, e_{n}$. Then there is a lattice non-regular tiling of $\mathbb{R}^{n}$ by $C$ if there is a homomorphism $\phi: \mathcal{M} \rightarrow G$, an Abelian group of order $2|V|$, so that the restriction of $\phi$ to $W=V \cup\left\{V+\frac{1}{2} e_{1}\right\}$ is a bijection and $\phi\left(e_{i}\right)$ is a generator of $G$ for some $i \geq 2$.

Proof: The homomorphism $\phi$ determines a tiling $\mathcal{T}=\{W+l ; l \in \mathcal{L}\}$ of $\mathcal{M}$ by $W$, where $\mathcal{L}=\operatorname{ker}(\phi)$ is a lattice. Clearly, $\mathcal{T}$ generates a tiling of $\mathbb{R}^{n}$ by the cluster $C$ of cubes centered in $V$. Indeed, split each cube in $C$ by a hyperplane orthogonal to $e_{1}$. Then we get a collection of $2|C|$ half-cubes centered at points of $W=V \cup\left\{V+\frac{1}{2} e_{1}\right\}$ in $\mathcal{M}$. Thus both a tiling $\mathcal{F}$ of $Z^{n}$ by $V$ and a tiling $\mathcal{F}^{\prime}$ of $\mathcal{M}$ by $W=V \cup\left\{V+\frac{1}{2} e_{1}\right\}$ they generate a tiling of $\mathbb{R}^{n}$ by the cluster $C$. The only difference between $\mathcal{F}$ and $\mathcal{F}^{\prime}$ is that while the centers of cubes of $\mathcal{F}$ are in $Z^{n}$, the centers of the half-cubes in $\mathcal{F}^{\prime}$ are in $\mathcal{M}$. To prove that $\mathcal{T}$ generates a non-regular tiling of $\mathbb{R}^{n}$ it suffices to show that there are $l, l^{\prime}$ in $\mathcal{L}$ so that $l_{1}$ is an integer while $l_{1}^{\prime}$ is of the form $m+0.5, m \in Z$, where $l_{1}$ and $l_{1}^{\prime}$ are the first coordinates of $l$ and $l^{\prime}$, respectively. Indeed, then the centers of cubes in $C+l$ will be at integer points, while the centers of cubes in $C+l^{\prime}$ will not. Since $\phi\left(e_{i}\right)$, for some $i \geq 2$, is a generator of $G$ then there are numbers $a, b \in Z$ so that $\phi\left(a e_{i}\right)=0$ and $\phi\left(\frac{1}{2} e_{1}+b e_{i}\right)=0$. Thus, the two vectors $l=a e_{i}, l^{\prime}=\frac{1}{2} e_{1}+b e_{i} \in \mathcal{L}$ have the required property.

\section{DiAMETER PERFECT LEE CODES}

This section contains main results of our paper. We focus on the existence and enumeration of diameter perfect Lee codes over $Z_{q}^{n}$ and $Z^{n}$. It is conjectured in [7] and [4], see Section 2 , that for $n \geq 3$ these codes exist only when $d=3$, 4 , with the exception of $\operatorname{DPL}(3,6)$ code constructed by Minkowski. We concentrate on linear codes as they are most important from the practical point of view. All values of $q$ for which there exists a linear $\operatorname{PL}(n, 1, q)$ code (=linear $\operatorname{DPL}(n, 3, q)$ code) have been found in [1]. Now we determine all values of $q$ for which there is a linear $\operatorname{DPL}(n, 4, q)$ code. Clearly, it suffices to determine all values $q$ for which there exists a linear non-periodic $\operatorname{DPL}(n, d, q)$ code as all other linear $\operatorname{DPL}(n, d, q)$ codes can be obtained by a periodic extension of a non-periodic one.

The following theorem claims that instead of investigating diameter perfect codes of $Z_{q}^{n}$ one can confine himself to codes in $Z^{n}$. The statement is intuitively clear and in some papers the authors take validity of analogous cases for granted. We will sketch its proof. We recall that a set $\mathcal{S} \subset Z_{*}^{n}$ is $p$-periodic, $p>0$, if $s \in \mathcal{S}$ iff $s+p e_{i} \in \mathcal{S}$ for all $i=1, \ldots, n$, and $p$ is the smallest positive number with the property.

Theorem 12: For $d$ is even, there is a linear $D P L(n, d, q)$ code if and only if there is a linear $\operatorname{DPL}(n, d) p$-periodic code with $p \mid q$.

Proof: We start with the following auxiliary statement.

Lemma. Let $\mathcal{L}$ be a linear diameter- $d$ perfect Lee code in $Z_{*}^{n}$ and $W$ be a double-sphere $D S_{n, r}$ with $r=\frac{d-2}{2}$. Then $\mathcal{T}=$ $\{W+l ; l \in \mathcal{L}\}$ is a lattice tiling of $Z_{*}^{n}$ by double-spheres.

First we prove this lemma. Assume that for $l \neq l^{\prime}$, there is $x \in$ $Z_{*}^{n}$ such that $x \in W+l \cap W+l^{\prime}$. Then there are $w, w^{\prime} \in W$ so that $x=w+l=w^{\prime}+l^{\prime}$. This in turn implies $\left|w-w^{\prime}\right|=$ $\left|l^{\prime}-l\right|$ which is a contradiction since $\left|l-l^{\prime}\right|=\rho_{L}\left(l, l^{\prime}\right) \geq d$ for any $l, l^{\prime} \in \mathcal{L}$ as $\mathcal{L}$ is a diameter- $d$ code while $\left|w-w^{\prime}\right|=$ $\rho_{L}\left(w, w^{\prime}\right)<d$ for any $w, w^{\prime} \in W$ because $W$ is an anticode of diameter $d-1$. To finish this part of the proof we need to show that $\bigcup_{l \in \mathcal{L}} W+l=Z_{*}^{n}$. This is obvious for $Z_{*}^{n}=Z_{q}^{n}$ as the space is finite and $\mathcal{L}$ is a transversal of a tiling by double-spheres. If $\mathcal{L}$ is a linear code then the tiling is a lattice tiling. Assume now that $\mathcal{L}$ is a linear $\operatorname{DPL}(n, d)$ code, and $\bigcup_{\mathcal{L}} W+l \varsubsetneqq Z^{n}$. Then the volume $V(\mathcal{L})$ of the lattice $\mathcal{L}$ would

$l \in \mathcal{L}$
be strictly bigger than the volume of the double-sphere $D S_{n, r}$ which is a contradiction as $\mathcal{L}$ is a transversal of a tiling of $Z^{n}$ by double-spheres. The proof of Lemma is complete.

Let $\mathcal{L}$ be a linear $\operatorname{DPL}(n, d, q)$ code. Then $\rho_{L}\left(l, l^{\prime}\right) \geq d$ for all $l, l^{\prime} \in \mathcal{L}$, and by the above lemma $T=\{W+l ; l \in$ $\mathcal{L}\}$ is a lattice tiling of $Z_{q}^{n}$ by double-spheres $D S_{n, r}, r=$ $\frac{d-2}{2}$. Consider an extension of $\mathcal{L}$ given by $\mathcal{L}^{\prime}=\left\{l^{\prime} ; l^{\prime}=\right.$ $l+\sum_{i=1}^{n} k_{i} q e_{i}$, where $l \in \mathcal{L}$, and $k_{i} \in Z$ for all $\left.i=1, . ., n\right\}$. It is a matter of technical routine to prove that $\mathcal{L}^{\prime}$ is a lattice as well with $\rho_{L}\left(l, l^{\prime}\right) \geq d$ for all $l, l^{\prime} \in \mathcal{L}^{\prime}$, of period $q$, and $\mathcal{T}^{\prime}=\left\{W+l^{\prime} ; l^{\prime} \in \mathcal{L}^{\prime}\right\}$ is a lattice tiling of $Z^{n}$. Therefore, $\mathcal{L}^{\prime}$ is a linear $D P L(n, d)$ code that is $p$-periodic for some $p \mid q$. On the other hand, let $\mathcal{L}$ be a linear $\operatorname{DPL}(n, d) q$-periodic code. Then, $\rho_{L}\left(l, l^{\prime}\right) \geq d$ for all $l, l^{\prime} \in \mathcal{L}$, and by our lemma, $T=\{W+l ; l \in \mathcal{L}\}$ is a lattice tiling of $Z^{n}$. Let $\mathcal{L}^{\prime}=$ $\mathcal{L} \cap Z_{q}^{n}$ be a restriction of $\mathcal{L}$ to $Z_{q}^{n}$. Clearly, $\rho_{L}\left(l, l^{\prime}\right) \geq d$ for every $l, l^{\prime} \in \mathcal{L}^{\prime}$. Further, as above, it is a matter of routine to 
verify that $\mathcal{L}^{\prime}$ is a lattice and $\left\{W+l ; l \in \mathcal{L}^{\prime}\right\}$ is a tiling of $Z_{q}^{n}$ by double-spheres $D S_{n, r}, r=\frac{d-2}{2}$. Thus, $\mathcal{L}^{\prime}$ is a linear $\operatorname{DPL}(n, d, q)$ code.

The previous theorem claims that instead of showing the existence of a distance- $d$ perfect linear Lee code over $Z_{q}^{n}$ (=a tiling of $Z_{q}^{n}$ by double-spheres) it suffices to prove the existence of a linear, $p$-periodic, $p \mid q$, distance- $d$ perfect Lee code over $Z^{n}$ (=a tiling of $Z^{n}$ by double-spheres). The difference in difficulty of proving the existence of a lattice tiling of $Z_{q}^{n}$ and of $Z^{n}$ might seem to be negligible. However, when looking for a tiling of $Z^{n}$ one has at its disposal a powerful construction based on groups homomorphism described in the previous section.

We are ready to determine all $q$ for which there exists a nonperiodic linear $\operatorname{DPL}(n, 4, q)$ code.

Theorem 13: Let $n=2^{\alpha} p_{1}^{\alpha_{1}} \ldots p_{k}^{\alpha_{k}}$ be the prime number factorization of $n$, where possibly $\alpha=0$, and $p_{i}>2$ for $i=1, \ldots, k$. Set $p=p_{1} \ldots p_{k}$. Then a linear non-periodic $\operatorname{DPL}(n, 4, q)$ code exists if and only if $q=2^{\beta} p_{1}^{\beta_{1}} \ldots p_{k}^{\beta_{k}}$, where $2 \leq \beta \leq \alpha+2$, and $1 \leq \beta_{i} \leq \alpha_{i}$. In particular, the smallest $q$ for which there exists a linear non-periodic $D P L(n, 4, q)$ code equals $4 p$.

Proof: Assume that there is a linear non-periodic $\operatorname{DPL}(n, 4, q)$ code. By Theorem 12 there is a linear $q$-periodic $D P L(n, d)$ code $\mathcal{L}$, and by the lemma stated in the proof of Theorem 12, $\mathcal{T}=\{V+l ; l \in \mathcal{L}\}$ is a lattice tiling of $Z^{n}$ by double-spheres $D S_{n, 1}$. As a copy $V$ of $D S_{n, 1}$ we choose $V=$ $\left\{ \pm e_{i}, \pm e_{i}+e_{1} ; i=1, \ldots, n\right\}$. Clearly, $|V|=4 n$. By Theorem 6 there is a homomorphism $\phi: Z^{n} \rightarrow G$, an Abelian group of order $4 n$, such that the restriction of $\phi$ to $V$ is a bijection. First we show that $4 p \mid q$. We note that $4 \mid \operatorname{ord}\left(\phi\left(e_{1}\right)\right)$. Indeed, consider the subgroup $H$ generated by $\phi\left(e_{1}\right)$. Then it is easy to check that for each $i, 1 \leq i \leq n,\left|\phi\left( \pm e_{i}, \pm e_{i}+e_{1}\right) \cap H\right|=0$ or 4 . Note that this is true also for $i=1$. Thus $4 \mid \operatorname{ord}\left(\phi\left(e_{1}\right)\right)$, which in turn implies $4 \mid q$, see Corollary 7 . Now we prove that $p \mid q$. Let $G=Z_{t_{1}} \times Z_{t_{2}} \times \ldots \times Z_{t_{s}}$, be the factorization of $G$, where possibly $s=1$; that is, possibly $G$ is a cyclic group. We recall that $n=2^{\alpha} p_{1}^{\alpha_{1}} \ldots p_{k}^{\alpha_{k}}$, so $4 n=2^{\alpha+2} p_{1}^{\alpha_{1}} \ldots p_{k}^{\alpha_{k}}$. We show that, for every $j=1, \ldots, k$, there is $i, 1 \leq i \leq n$, depending only on $j$ so that $p_{j} \mid \operatorname{ord}\left(\phi\left(e_{i}\right)\right)$. By Corollary 7 this will in turn imply that $p \mid q$. As $4 n=t_{1} \times \ldots \times t_{s}$, there is $t_{i}$ so that $p_{j} \mid t_{i}$; wlog assume $p_{j} \mid t_{1}$. Let $g=\phi\left(e_{1}\right)=\left(g_{1}, g_{2} \ldots, g_{s}\right)$. We consider two cases. If $p_{j} \nmid g_{1}$, then $p_{j} \mid \operatorname{ord}(g)$ and we are done. Otherwise, for $p_{j} \mid g_{1}$, consider the element $f=(1,0, \ldots, 0)$. Since the restriction of $\phi$ to $V$ is a bijection, there is $i$ so that $f \in \phi\left(\left\{ \pm e_{i}, \pm e_{i}+e_{1}\right\}\right.$. If $f=\phi\left(e_{i}\right)$ or $f=\phi\left(e_{i}\right)^{-1}$ we are done as $p_{j} \mid \operatorname{ord}\left(\phi\left(e_{i}\right)\right)=t_{1}$. Finally we are left with two cases, either $f=\phi\left(e_{i}+e_{1}\right)=\phi\left(e_{1}\right)+g$ or $f=\phi\left(-e_{i}+e_{1}\right)=-\phi\left(e_{i}\right)+g$ for some $i$. In the former case, $\phi\left(e_{i}\right)=f-g=\left(1-g_{1},-g_{2}, \ldots,-g_{s}\right)$, in the latter case $\phi\left(e_{i}\right)=g-f=\left(-1+g_{1}, g_{2}, \ldots, g_{s}\right)$. As $p_{j} \mid g_{1}$ and $p_{j} \mid t_{1}$ we have $p_{j} \nmid \pm\left(1-g_{1}\right)$, that is $p_{j} \mid \operatorname{ord}\left(\phi\left(e_{i}\right)\right)$ in both cases. Thus we proved that $p_{j} \mid q$, which implies that $p \mid q$. In aggregate, $4 p \mid q$. It is obvious that $q \leq 4 n$. Indeed, by Corollary $7 \mathcal{T}$ is $q$-periodic where $q=$ l.c.m. $\left(\operatorname{ord}\left(\phi\left(e_{1}\right)\right), \ldots, \operatorname{ord}\left(\phi\left(e_{n}\right)\right)\right)$. As the order of each element divides the order of the group also $q$ does, so $q \leq \operatorname{ord}(G)=4 n$, which in aggregate implies that $q=2^{\beta} p_{1}^{\beta_{1}} \ldots p_{k}^{\beta_{k}}$, where $2 \leq \beta \leq \alpha+2$, and $1 \leq \beta_{i} \leq \alpha_{i}$.

Now we show that there is a linear non-periodic $D P L(n, 4, q)$ code for each $q=2^{\beta} p_{1}^{\beta_{1}} \ldots p_{k}^{\beta_{k}}$, where $2 \leq \beta \leq \alpha+2$, and $1 \leq \beta_{i} \leq \alpha_{i}$. By Theorems 2 and 12 , it suffices to construct a $q$-periodic, lattice tiling $\mathcal{T}=\{V+l ; l \in \mathcal{L}\}$ of $Z^{n}$ by doublespheres so that, for any $l \in \mathcal{L}$, the Lee weight $|l|$ of $l$ is even. This tiling $\mathcal{T}$ will be constructed by means of Theorem 6 Hence it suffices to find a homomorphism $\phi: Z^{n} \rightarrow G$, where $G$ is an Abelian group of order $4 n$, so that the restriction of $\phi$ to a $V$ is a bijection. To guarantee that $\mathcal{T}$ is $q$-periodic, we choose $G$ so that $\operatorname{ord}(g) \mid q$ for each $g \in G$, see Corollary 7 , and we choose $\phi\left(e_{1}\right)$ so that $\operatorname{ord}\left(\phi\left(e_{1}\right)\right)=q$.

To have a group $G$ of order $4 n$ with the property $\operatorname{ord}(g) \mid q$ for each $g \in G$ we set $G=Z_{t_{1}} \times Z_{t_{2}} \times \ldots \times Z_{t_{s}}$, where $t_{1}=q$, and $t_{i}=2^{\gamma} p_{1}^{\gamma_{1}} \ldots p_{k}^{\gamma_{k}}$, where $\gamma, \gamma_{j} \in\{0,1\}$ for all $i>1,1 \leq j \leq k$.

The homomorphism $\phi$ is defined by choosing $g_{i}:=\phi\left(e_{i}\right)$. First we set $g_{1}=(1,0, \ldots, 0)$ and $g_{i}=(2 i-1,0, \ldots, 0)$ for $2 \leq i \leq \frac{q}{4}$. It is easy to check that $\phi\left(\left\{ \pm e_{i}, \pm e_{i}+e_{1} ; i=\right.\right.$ $\left.1, \ldots, \frac{t}{4}\right\}=Z_{q} \times\{0\} \times \ldots \times\{0\}$.

Similarly, for $b=\left(0, b_{2}, \ldots, b_{s}\right) \in G, b \neq 0$, with $\operatorname{ord}(b)=2$, we set $g_{i+j}=\left(2 i-1, b_{2}, \ldots, b_{s}\right), i=1, \ldots, \frac{q}{4}$, for a suitable fixed $\dot{j}$. Clearly, $\phi\left(\left\{ \pm e_{i+j}, \pm e_{i+j}+e_{1} ; i=1, \ldots, \frac{q}{4}\right\}=Z q \times\right.$ $\left\{b_{2}\right\} \times \ldots \times\left\{b_{n}\right\}$.

Finally, if $b=\left(0, b_{2}, \ldots, b_{s}\right) \in G, b \neq 0$, with $\operatorname{ord}(b) \neq 2$, we associate $\frac{q}{2}$ dimensions with the elements $b$ and $b^{-1}$ by $g_{i+j}=\left(2 i-1, b_{2}, \ldots, b_{s}\right), i=1, \ldots, \frac{q}{2}$, where $j$ is a suitable fixed number. Then $\phi\left(\left\{ \pm e_{i+j}, \pm e_{i+j}+e_{1} ; i=1, \ldots, \frac{q}{2}\right\}=\right.$ $Z_{q} \times\left\{b_{2}\right\} \times \ldots \times\left\{b_{n}\right\} \cup Z_{q} \times\left\{-b_{2}\right\} \times \ldots \times\left\{-b_{n}\right\}$. It is easy to see that in aggregate $\phi(V)=G$. So the restriction of the mapping $\phi$ to $V$ is a bijection. Thus we proved that there exists a lattice tiling $\mathcal{T}$ of $Z^{n}$ by double-spheres that is $q$-periodic.

Denote the lattice in the tiling $\mathcal{T}$ by $\mathcal{L}$. We will prove that, for each $l \in \mathcal{L}$, the Lee weight $|l|$ is even, which in turn implies, by Theorem 2 , that $\mathcal{L}$ is a linear $\operatorname{DPL}(n, d)$ code. To prove it we will construct a vector basis $v_{i}, i=1, \ldots, n$, of $\mathcal{L}$ whose each element is of even weight. First we set $v_{1}=q e_{1}$. As $q$ is even $\left|v_{1}\right|=q$ is even, and $\phi\left(v_{1}\right)=(q, 0, \ldots, 0)=(0, \ldots, 0)$; i.e. $v_{1} \in \operatorname{ker}(\phi)=\mathcal{L}$. Further, we set $v_{i}=(2 i-1) e_{1}-e_{i}$ for $i=2, \ldots, \frac{q}{4}$. Also in this case we get $\phi\left(v_{i}\right)=(2 i-1,0, . ., 0)-$ $(2 i-1,0, \ldots, 0)=(0, \ldots, 0)$ and $\left|v_{i}\right|=2 i-2$. Now, for each $j=2, \ldots, s$ there is $m_{j}$ so that $\phi\left(e_{m_{j}}\right)=(1,0, \ldots 0,1,0, \ldots 0)$ with $j$-th coordinate equal to 1 . We set $v_{m_{j}}=t_{j} e_{1}-t_{j} e_{m_{j}}$. Again, as before, $\phi\left(v_{m_{j}}\right)=(0, \ldots, 0)$ and $\left|v_{m_{j}}\right|=2 t_{j}$ is even. Finally, if $\phi\left(e_{i}\right)=\left(b_{1}, b_{2}, \ldots b_{s}\right)$, where $i>\frac{q}{4}, i \neq m_{j}, j=$ $2, \ldots, s$, then we set $v_{i}=\left(b_{1}-b_{2}-\ldots-b_{s}\right) e_{1}+b_{2} e_{m_{2}}+$ $\ldots+b_{s} e_{m_{s}}-e_{i}$. We get $\phi\left(v_{i}\right)=\left(b_{1}-b_{2}-\ldots-b_{s}, 0, \ldots, 0\right)+$ $\left(b_{2}, b_{2}, 0, \ldots, 0\right)+\left(b_{3}, 0, b_{3}, 0, \ldots, 0\right)+\ldots+\left(b_{s}, 0, \ldots, 0, b_{s}\right)-$ $\left(b_{1}, b_{2}, \ldots b_{s}\right)=(0, \ldots, 0)$ and $\left|v_{i}\right|=b_{1}+2\left(b_{2}+\ldots+b_{s}\right)+1$ 


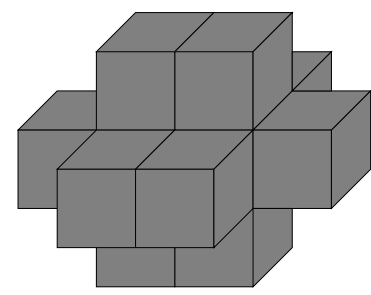

Fig. 5: 3-double-cross

which is even as $b_{1}$ is always odd, see definition of $g_{i}=$ $\phi\left(e_{i}\right)$. Thus we showed that, for all $i=1, \ldots, n, v_{i}$ belongs to $\operatorname{ker} \phi=\mathcal{L}$ and $\left|v_{i}\right|$ is even. It is not difficult to see that $v_{i}, i=1, \ldots, n$, are independent vectors. Let $A$ be a matrix with $v_{i}$ being its $i$-th row. After permuting columns of $A$ so that the columns $m_{j}, j=2, \ldots, s$, become columns indexed $\frac{q}{4}+1, \ldots, \frac{q}{4}+s$, we get a lower triangular matrix with its diagonal equal to $t_{1},-1, \ldots,-1,-t_{2},-t_{3}, \ldots,-t_{s},-1, \ldots,-1$. Therefore $|\operatorname{det} A|=t_{1} t_{2} \ldots t_{s}=4 n$, which in turn implies that $v_{i}$ 's form a basis with the required properties. The proof is complete.

A cluster consisting of $2 n+1$ unit cubes centered at points of Lee sphere $S_{n, 1}$ is called the $n$-cross. By the $n$-doublecross we understand a cluster of $4 n$ unit cubes centered at points of double-sphere $D S_{n, 1}$, see Fig. 5 for 3 -double-cross. Szabo [26] proved that there is a non-regular lattice tiling of $\mathbb{R}^{n}$ by $n$-crosses if and only if $2 n+1$ is not a prime. The nonexistence of such lattice tiling for $2 n+1$ being a prime follows from Redei's decomposition theorem see [20]. We show that, for some $n$, there is a non-regular lattice tiling of $\mathbb{R}^{n}$ by $n$ double-crosses. At the first glance it seems that non-regular tilings are not useful in coding theory. However, we will show that they are instrumental for enumerating $D P L(n, 4)$ codes.

Theorem 14: Let $n>1$ be a natural number that is not a power of 2. Then there exists a non-regular lattice tiling of $\mathbb{R}^{n}$ by $n$-double-crosses.

Proof: As above we use the double sphere $V=$ $\left\{ \pm e_{i}, \pm e_{i}+e_{1} ; i=1, \ldots, n\right\}$. By Theorem 11 it suffices to find an Abelian group $G$ of order $8 n$ and a homomorphism $\phi: \mathcal{M} \rightarrow G$ so that the restriction of $\phi$ to $W=V \cup\left\{V+\frac{1}{2} e_{1}\right\}$ is a bijection and $\phi\left(e_{i}\right)$ is a generator of $G$ for some $i>1$. Since $n$ is not a power of 2 , then $n$ can be written in the form $n=2^{t}(2 k+1), k>0$. As $G$ we choose the cyclic group $Z_{8 n}$. We set

$\phi\left(\frac{1}{2} e_{1}\right)=2 k+1$, and, for $t>0, \phi\left(e_{i}\right)=(4 i-2)(2 k+$ $1), i=2, \ldots, 2^{t}$. This way $\phi\left(\bigcup_{i-1}^{2^{t}}\left\{ \pm e_{i}, \pm e_{i}+e_{1}\right\} \cup\left\{\left\{ \pm e_{i}, \pm e_{i}+\right.\right.\right.$ $\left.\left.\left.e_{1}\right\}+\left\{\frac{1}{2} e_{1}\right\}\right\}\right)=\{s ; 0 \leq s<8 n, s \equiv 0(\bmod )(2 k+1)\}$.

For $j=1, \ldots, k$, we set $\phi\left(e_{i+c_{j}}\right)=j+4(i-1)(2 k+1)$ for $i=1, \ldots, 2^{t+1}$, where $c_{j}=2^{t}+(j-1) 2^{t+1}$. For fixed $j$ we have $\phi\left(\bigcup\left\{ \pm e_{i}, \pm e_{i}+e_{1}\right\} \cup\left\{\left\{ \pm e_{i}, \pm e_{i}+e_{1}\right\}+\left\{\frac{1}{2} e_{1}\right\}\right\}\right)$, where $\left.2^{t}+(j-1) 2^{t+1}+1 \leq i \leq 2^{t}+j 2^{t+1}\right)=\{s ; 0 \leq s<8 n$, $s \equiv \pm j(\bmod )(2 k+1)\}$. Thus, in aggregate, $\phi(V)=G$. To finish the proof it is sufficient to notice that $\phi\left(e_{1+2^{t}}\right)=1$, a generator of $G$. The proof is complete.

As mentioned in the introduction the double-sphere $D S_{3.1}$ can be seen as the union of two Lee spheres $S_{3,1}$ with centers at distance 1 . Therefore it is somewhat surprising that there exists a non-regular tiling $\mathcal{T}$ of $\mathbb{R}^{3}$ by 3 -double-cross although there is no non-regular tiling of $\mathbb{R}^{3}$ by 3 -crosses. A detailed description of the tiling $\mathcal{T}$ will be provided in the proof of the following theorem that enumerates $\operatorname{DPL}(n, 4)$ codes for all $n \geq 2$. We recall that two tilings $\mathcal{T}=\{V+l ; l \in \mathcal{L}\}$ and $\mathcal{T}^{\prime}=\left\{V+l ; l \in \mathcal{L}^{\prime}\right\}$ are called congruent (two codes $\mathcal{L}$ and $\mathcal{L}^{\prime}$ are called isomorphic) if there exists a linear distancepreserving bijection $Z^{n} \rightarrow Z^{n}$ that maps $\mathcal{L}$ on $\mathcal{L}^{\prime}$.

Theorem 15: For each $n \geq 2$, there are $2^{\aleph_{0}}$ non-isomorphic $D L P(n, 4)$ codes.

Proof: First of all we show that the total number of $D P L(n, 4)$ codes is at most $2^{\aleph_{0}}$. Each $\operatorname{DPL}(n, 4)$ code is a subset of $Z^{n}$ of cardinality $\aleph_{0}$. This in turn implies that the total number of $\operatorname{DLP}(n, 4)$ codes in $Z^{n}$ is at most the number of ways how to choose $\aleph_{0}$ points in $Z^{n}$; thus it is at most $\left(\aleph_{0}\right)^{\aleph_{0}}=2^{\aleph_{0}}$. Therefore, there are at most $2^{\aleph_{0}}$ $D P L(n, 4)$ codes. To prove the statement we will construct $2^{\aleph_{0}}$ non-isomorphic $D P L(n, 4)$ codes. Etzion [7] proved that there are $2^{\aleph_{0}}$ non-isomorphic $\operatorname{DPL}(n, 4)$ codes for $n$ being a power of 2 . So we are left with the case when $n$ is not a power of 2 .

Let $n=2^{t}(2 k+1)$. By Theorem 14 there exists a non-regular lattice tiling $\mathcal{T}=\{W+l ; l \in \mathcal{L}\}$ of $\mathbb{R}^{n}$ by double-crosses, where $W$ is the $n$-double-cross comprising unit cubes centered at words in $V=\left\{ \pm e_{i}, \pm e_{i}+e_{1} ; i=1, \ldots, n\right\}$. For the purpose of this proof it is convenient to know a basis of the lattice $\mathcal{L}$. Let $j=2^{t}+1$. Consider vectors $v_{i}, i=1, \ldots, n$, given by $v_{1}=-\frac{1}{2} e_{1}+(2 k+1) e_{j}, v_{j}=8 n e_{j}$, and $v_{i}=\phi\left(e_{i}\right) e_{j}-e_{i}$, for $2 \leq i \leq n, i \neq j$. It is easy to check that $\phi\left(v_{i}\right)=0$ as $\phi\left(e_{j}\right)=1$ and $\phi\left(\frac{1}{2} e_{1}\right)=2 k+1$, see the proof of Theorem 14 , i.e., $v_{i} \in \mathcal{L}$ for all $i=1, \ldots, n$. Let $A$ be a matrix whose $i$-th row is the vector $v_{i}$. Transpose the first and the $j$-th column and the first and the $j$-th row of $A$. An element of the resulting matrix $A^{\prime}$ is non-zero if and only if it is in the first column or it is a diagonal element of $A^{\prime}$. Thus $v_{i}$ 's are independent vectors. Moreover, $\left|\operatorname{det}\left(A^{\prime}\right)\right|$ equals the product of its diagonal elements; thus $|\operatorname{det}(A)|=8 n\left(-\frac{1}{2}\right)(-1)^{n-2}=4 n$. Therefore $v_{i}, i=1, \ldots, n$, constitute a basis of $\mathcal{L}$.

Consider a relation $R$ on $\mathcal{L}$ given by $m R n$ if for their first coordinates $m_{1}, n_{1}$ it is $m_{1}-n_{1} \in Z$. It is easy to check that $R$ is an equivalence relation on $\mathcal{L}$ with two equivalence classes $R_{1}=\left\{l ; l \in L, l_{1} \in Z\right\}$ and $R_{2}=\left\{l ; l \in \mathcal{L}, l_{1}=k+0.5, k \in\right.$ $Z\}$ where $l_{1}$ is the first coordinate of $l$. For $i=1,2$, set $K_{i}=\bigcup_{l \in \mathcal{R}_{i}}\{W+l\}$. Clearly, $\mathcal{K}_{1}$ and $\mathcal{K}_{2}$ form a partition of $\mathbb{R}^{n}$. It is well known, see e.g. [25], [26], that $R_{1}$ is a sublattice of $\mathcal{L}$ and that $R_{2}$ is a coset of $\mathcal{L} / R_{1}$; thus $R_{2}=R_{1}+v_{1}$ and also $\mathcal{K}_{2}=\mathcal{K}_{1}+v_{1}$. Moreover, both $\mathcal{K}_{1}$ and $\mathcal{K}_{2}$ constitute 


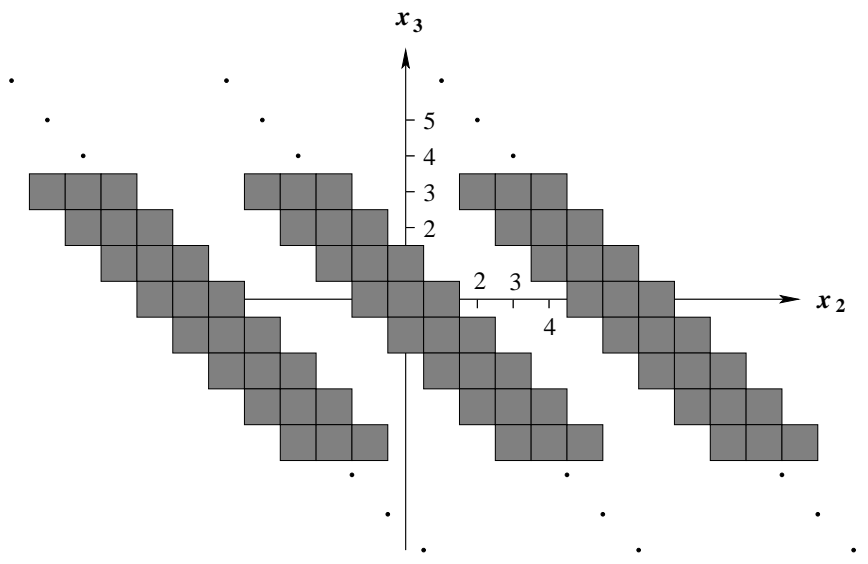

Fig. 6: The intersection of $x_{2} x_{3}$-plane with $K_{1}$

prisms along $x_{1}$-axis. That is, if a point $x \in \mathcal{K}_{i}, i \in\{1,2\}$ then the whole line parallel to $x_{1}$-axis passing through $x$ belongs to $\mathcal{K}_{i}$. As a very important consequence we get that tiles in $\mathcal{K}_{2}$ can be shifted along $x_{1}$-axis independently of tiles in $\mathcal{K}_{1}$. For example, shifting $\mathcal{K}_{2}$ by $0.5 e_{1}$ produces a tiling $\mathcal{S}$ of $\mathbb{R}^{n}$ by $n$-double-crosses with all their cubes centered at integer points. It is easy to show that $\mathcal{S}$ is not a lattice tiling but it is a periodic tiling.

Now we show that in fact both $\mathcal{K}_{1}$ and $\mathcal{K}_{2}$ consist of infinitely many connectivity (in the topological sense) components. For the reader's convenience we start with $n=3$. Then the basis of $\mathcal{L}$ comprises vectors $v_{1}=-\frac{1}{2} e_{1}+3 e_{2}, v_{2}=24 e_{2}$, and $v_{3}=13 e_{2}-e_{3}$. Note that cubes centered at $\left\{O, \pm e_{2}\right\}$ belong to $\mathcal{K}_{1}$ as $V+O=V \in \mathcal{K}_{1}$. Further, as $\mathcal{K}_{1}$ is a prism along $x_{1^{-}}$ axis, and $2 t v_{1} \in R_{1}$ for all $t \in Z$, we have that cubes centered at $\left\{O, \pm e_{2}\right\}+6 t e_{2} \in \mathcal{K}_{1}$ for all $t \in Z$. Similarly, all cubes centered at $\left\{O, \pm e_{2}\right\}+e_{2}-e_{3} \in \mathcal{K}_{1}$ as $4 v_{1}+v_{2}=-2 e_{1}+e_{2}-$ $e_{3} \in R_{1}$. Thus all cubes centered at $\left\{O, \pm e_{2}\right\}+6 t e_{2}+s\left(e_{2}-\right.$ $\left.e_{3}\right) \in \mathcal{K}_{1}$ for all $s, t \in Z$; see Fig. 6 , where the intersection of the $x_{2} x_{3}$-plane with $\mathcal{K}_{1}$ is shaded. The rest of the plane belongs to the intersection with $\mathcal{K}_{2}$ as $\mathcal{K}_{2}=\mathcal{K}_{1}+v_{1}$ and both $\mathcal{K}_{i}$ s are prisms along $x_{1}$-axis. Thus we proved that both $\mathcal{K}_{1}$ and $\mathcal{K}_{2}$ comprise infinitely many components. In particular, if $B_{m}, m \in Z$, is the collection of all components in $\mathcal{K}_{2}$ then the point $3(2 m+1) e_{2} \in B_{m}$. The proof for $n>3, n$ not a power of 2 , would be very similar although technically more involved. In this case the points $(2 k+1)(2 m+1) e_{2}$ belong to distinct connectivity components $B_{m}$ of $\mathcal{K}_{2}, m \in Z$; we recall that $n=2^{t}(2 k+1)$.

The connectivity components of $\mathcal{K}_{2}$ can be shifted along $x_{1}$ axis independently of each other and on $\mathcal{K}_{1}$. This property of $\mathcal{K}_{2}$ will be used to construct $2^{\aleph_{0}}$ non-isomorphic $\operatorname{DPL}(n, 4)$ codes. Let $r \in(0,1)$ be an irrational number, $r=0 . r_{1} \ldots r_{k} \ldots$ be its binary representation. A tiling $\mathcal{T}_{r}$ will be obtained from the tiling $\mathcal{T}$ by translating connectivity components $B_{m}$ of $\mathcal{K}_{2}$ as follows: If $m \leq 0$ or $r_{m}=0$ then the component $B_{m}$ will be shifted by $-\frac{1}{2} e_{1}$, otherwise it will be shifted by $\frac{1}{2} e_{1}$. Clearly all double-crosses in $\mathcal{T}_{r}$ are centered at integer points. In other words, $\mathcal{T}_{r}=\left\{W+l ; l \in \mathcal{L}^{\prime}\right\}$ is a tiling such that all coordinates of any $l \in \mathcal{L}^{\prime}$ are integers. Such tiling will be called a $Z$-tiling. Obviously, for any two irrational numbers $r$ and $s$, the corresponding tilings $\mathcal{T}_{r}$ and $\mathcal{T}_{s}$ are distinct; we recall that two tilings $\{W+l ; l \in \mathcal{L}\}$ and $\left\{W+l ; l \in \mathcal{L}^{\prime}\right\}$ are called distinct if $\mathcal{L}$ and $\mathcal{L}^{\prime}$ are distinct. So we have produced a family $\mathcal{S}=\left\{\mathcal{T}_{r} ; r\right.$ is an irrational number in $\left.(0,1)\right\}$ of $2^{\aleph_{0}}$ distinct $Z$-tilings of $\mathbb{R}^{n}$ by double-crosses. Each $Z$-tiling of $\mathbb{R}^{n}$ by double-crosses induces a tiling of $Z^{n}$ by double-spheres. So the family $\mathcal{S}$ induces a family $\mathcal{S}^{\prime}$ of $2^{\aleph_{0}}$ distinct tilings of $Z^{n}$ by double-spheres $V=\left\{ \pm e_{i}, \pm e_{i}+e_{1} ; i=1, \ldots, n\right\}$. Clearly $O \in \mathcal{L}$ for each tiling $\mathcal{T}=\{V+l ; l \in \mathcal{L}\}$, that is $V \in \mathcal{T}$ for each tiling $\mathcal{T}$ in $\mathcal{S}$. To each tiling $\mathcal{T}=\{V+l ; l \in \mathcal{L}\}$ in $\mathcal{S}^{\prime}$ we assign a set $\mathcal{T}_{N}=:\{y ; y$ is a center of $V+l, l \in \mathcal{L}$, and $|y|$ is even $\}=\{l ;|l|$ is even, $l \in \mathcal{L}\} \cup\left\{l+e_{1} ;|l|\right.$ is odd, $\left.l \in \mathcal{L}\right\}$, where $|x|=\rho_{L}(x, O)$ is the Lee weight of $x \in Z^{n}$. It was showed in the proof of Theorem 2] that $\mathcal{T}_{N}$ is a $D P L(n, 4)$ code. It is easy to see that if $\mathcal{T}$ and $\mathcal{T}^{\prime}$ are distinct tilings in $\mathcal{S}^{\prime}$ then the induced codes $\mathcal{T}_{N}$ and $\mathcal{T}_{N}^{\prime}$ are distinct as well. Thus, we have constructed a family $\mathcal{S}^{\prime \prime}$ of $2^{\aleph_{0}}$ distinct $D P L(n, 4)$ codes. As each code in $\mathcal{S}^{\prime \prime}$ is isomorphic to at most $\aleph_{0}$ codes in $\mathcal{S}^{\prime \prime}$ (we recall that two codes $\mathcal{T}$ and $\mathcal{T}^{\prime}$ are isomorphic if there exists a linear distance preserving bijection mapping $\mathcal{T}$ onto $\left.\mathcal{T}^{\prime}\right) \mathcal{S}^{\prime \prime}$ has to contain a set of $2^{\aleph_{0}}$ non-isomorphic $D P L(n, 4)$ codes. The proof is complete.

As an easy consequence we get:

Corollary 16: For each $n \geq 2$, the number of non-periodic $\operatorname{DPL}(n, 4)$ codes is $2^{\aleph_{0}}$.

Proof: It is easy to see that for each $p \in Z$ there are at most finitely many $p$-periodic $D P L(n, 4)$ codes. Therefore, in aggregate, there are at most $\aleph_{0}$ periodic $D P L(n, 4)$ codes. As there are in total $2^{\aleph_{0}} D P L(n, 4)$ codes, $2^{\aleph_{0}}$ of them have to be non-periodic.

We note that Szabo [26] constructed non-regular lattice tilings of $\mathbb{R}^{n}$ by $n$-crosses if $2 n+1$ is a prime. The non-existence of such lattice tiling for $2 n+1$ being a prime follows from a result of Redei [20] on factorization of Abelian groups. We have constructed a lattice non-regular tiling of $R^{n}$ by $n$-doublecross for all $n$ that are not a power of 2 . We believe that there are no non-regular tilings of $R^{n}$ by $n$-double-cross but at the moment we are not able to prove it.

\section{DECODING ALGORITHM FOR LINEAR PERFECT LEE CODES}

In this section we design an algorithm for decoding a linear $D P L(n, d)$ code. The computational complexity of this algorithm is linear in $n$ for $d=3,4$, and its complexity is $O(\log d)$ for $D P L(2, d)$ codes. We recall that $D P L(n, d)$ codes are believed not to exists for $n \geq 3$ and $d>4$. We stress that this algorithm can be applied to all perfect Lee codes as diameter perfect Lee codes include also perfect errorcorrecting codes. The efficiency of the algorithm is achieved thanks to the representation of linear codes by means of a tiling constructed through a group homomorphism. We design the algorithm for a linear $D P L(n, d)$ code. In case of a linear $D P L(n, d, q)$ code it suffices to realize that, by Theorem 
12. the code is a restriction of a $D P L(n, d)$ code and the corresponding algorithm would be nearly identical.

Let $\mathcal{L}$ be a linear $\operatorname{DPL}(n, d)$ code. Theorem 6 implies that there is a homomorphism $\phi: Z^{n} \rightarrow(G, \circ)$, where $G=Z^{n} / \mathcal{L}$ is an Abelian group of order $|W|, W$ is the anticode of diameter $d-1$ of maximum size, and that the restriction of $\phi$ to $W$ is a bijection. Denote by $f: G \rightarrow W$ the function inverse to the restriction of $\phi$ to $W$.

Assume that we receive a word $a \in Z^{n}$. First we find the element $g=\phi(a)$ of the group $G$. If $g=e$, the neutral element in $G$, we are done as $a$ is a codeword in $\mathcal{L}$. In general the corresponding codeword $l \in \mathcal{L}$ is given by $l=a-f(g)=$ $a-f(\phi(a))$. Indeed, $\mathcal{T}=\{W+l ; l \in \mathcal{L}\}$ is a tiling of $Z^{n}$ by $W$. Thus, each element $a \in Z^{n}$ can be written in a unique way as $a=l+w$, where $l \in \mathcal{L}$ is the sought codeword and $w \in W$. Hence, $l=a-w=a-f(g)=a-f(\phi(a))$.

Hence the complexity of the algorithm is in fact the number of operations needed for calculation of $f(\phi(a))$. We note that the number of operations in the calculation of $\phi(a)$ is linear in $n$ since $\phi(a)=\phi\left(\left(a_{1}, \ldots, a_{n}\right)\right)=\phi\left(e_{1}\right)^{a_{1}} \circ \ldots \circ \phi\left(e_{n}\right)^{a_{n}}$, where the values $\phi\left(e_{i}\right), i=1, \ldots, n$, will be stored. Now we bound from above the number of operations needed for the calculation of the values of the function $f$ for any element $g$ in the group $G$. The values of the function $f$ need to be calculated only once and therefore time needed for obtaining these values is not included in the complexity of the decoding algorithm. The values of $f$ will be stored in a tabular way and we will evaluate time needed to find the value of $f(g)$ in the table for given $g \in G$. The size of the table is the order of the group $G$, which equals $|W|$. It is known, see [7], that the volume of the double-sphere is

$$
\left|D S_{n, r}\right|=\sum_{i=0}^{\min \{n-1, r\}} 2^{i+1}\left(\begin{array}{c}
n-1 \\
i
\end{array}\right)\left(\begin{array}{c}
r+1 \\
i+1
\end{array}\right)
$$

The volume of the sphere $S_{n, r}$ is smaller than the volume of $D S_{n, r}$ therefore we find the bound only for the doublesphere $D S_{n, r}$. However, we can find the required value of $f$ in the table in an efficient way. As $G$ is an Abelian group, $G$ can be written as a direct product of cyclic groups $Z_{q_{1}} \times$ $\ldots \times Z_{q_{s}}$. We store values of $f$ in the lexicographic order; that is, first comes value of $f$ at $(0,0, \ldots, 0,0)$, then value of $f$ at $(0,0, \ldots, 0,1)$, then at $(0,0, \ldots, 2)$, etc. The rank $r$ of an element $a=\left(a_{1}, \ldots, a_{s}\right)$ in this order is

$$
r(a)=1+a_{s}+\sum_{i=1}^{s-1} a_{i} \prod_{j=i+1}^{s} q_{j}
$$

Hence, the rank $r(a)$ can be calculated in time linear with $s$ as from (2) it follows

$$
\begin{aligned}
r(a)= & 1+a_{s}+ \\
& q_{s}\left(a_{s-1}+q_{s-1}\left(a_{s-2}+q_{s-2}\left(a_{s-3}+\ldots+\right.\right.\right. \\
& \left.\left.\left.q_{3}\left(a_{2}+q_{2}\left(a_{1}\right)\right) \ldots\right)\right)\right)
\end{aligned}
$$

The maximum value of $s$ is achieved when $G=Z_{2} \times Z_{2} \times \ldots \times$ $Z_{2}$. Thus the maximum value of $s=\log |W|=\log \left|D S_{n, r}\right|$. For $n=2$ we get $\left|D S_{2, r}\right|=2(r+1)^{2}$, thus $s=O(\log d)$ where $d=2 r+2$. For $d=4$ it is $\left|D S_{n, 1}\right|=4 n$, thus $s=\log n$. In aggregate, the complexity of the algorithm for $D P L(n, d), d=3,4$, codes is $\Theta(n)$, and its complexity is $O(\log d)$ for $D P L(2, d)$ codes.

\section{REFERENCES}

[1] B. AlBdaiwi, P. Horak, and L. Millazo, Enumerating and decoding perfect linear Lee codes, Designs, Codes, and Cryptography 52 (2009), 155-162.

[2] R. Ahlswede, H. K. Audinian, and L. H. Khachatrian, On perfect codes and related concepts, Designs, Codes and Cryptography, 22(2001), 221237.

[3] R. Ahlswede and V. Blinovsky, Lectures on Advances in Combinatorics, Springer-Verlag, 2008.

[4] C. Araujo, I. Dejter, and P. Horak, A generalization of Lee codes, 3rd International Castle Meeting on Coding Theory and Applications,J. Borges and M. Villanueva (eds.), 2011, 25-30.

[5] J. Astola, On perfect codes in the Lee metric, Ann. Univ. Turku (A)1, 176, (1978), 56pp.

[6] S. I. Costa, M. Muniz, E. Agustini, and R. Palazzo, Graphs, tessellations, and perfect codes on flat tori, IEEE Trans. Inform. Th. 50 (2004), 23632377.

[7] T. Etzion, Product constructions for perfect Lee Codes, to appear in IEEE Trans. Inform. Th.

[8] S. W. Golomb and L. R. Welsh, Perfect codes in the Lee metric and the packing of polyominos, SIAM J. Applied Math. 18 (1970), 302-317.

[9] S. Gravier, M. Mollard, and Ch. Payan, On the existence of threedimensional tiling in the Lee metric, European J. Combinatorics 19 (1998), 567-572.

[10] W. Hamaker and S. K. Stein, Combinatorial packing of $R^{n}$ by certain error spheres, IEEE Trans. Inform. Th. 30 (1984), 364-368.

[11] D. Hickerson and S. K. Stein, Abelian groups and packings by semicrosses, Pacific J. Mathematics 122(1986), 96-109.

[12] P. Horak, On Perfect Lee Codes, Discrete Mathematics 309 (2009), 5551-5561.

[13] P. Horak, Tilings in Lee Metric, European J. Combinatorics 30(2009), 480-489.

[14] P. Horak, Error-correcting codes and Minkowski's conjecture, Tatra Mt. Math. Publ. 45 (2010), 37-49.

[15] P. Horak and B. AlBdaiwi, Non-periodeic tilings of $\mathbb{R}^{n}$ by crosses, Discrete \& Computational Geometry 47 (2012), 1-16.

[16] C. Y. Lee, Some properties on nonbinary error-correcting code, IRE Transactions on Information Th. 4(1958), 72-78

[17] H. Minkowski, Dichteste gitterförmige Lagerung kongruenter Korper, Nachrichten Ges. Wiss. Gottingen (1904), 311-355.

[18] E. Molnár, Sui Mosaici dello spazio de dimensione n, Atti della Accademia Nazionale dei Lincei, Rend. Sc. Fis. Mat. e Nat. 51(1971), 177-185.

[19] K. A. Post, Nonexistence Theorem on Perfect Lee Codes over Large Alphabets, Information and Control 29(1975), 369-380.

[20] L. Redei, Neuer Beweis des Hajósschen Satzes über die endlichen Abelschen Gruppen, Acta Math. Acad. Sci. Hungar. 6 (1955), 27-40.

[21] S. Špacapan, Non-existence of face-to-face four dimensional tiling in the Lee metric, European J. Combinatorics 28 (2007), 127-133.

[22] M. Schwartz, Quasi-cross lattice tilings with applications to flash memory, submitted.

[23] S. K. Stein, Factoring by subsets, Pacific J. Mathematics 22(1967), 523541.

[24] S. K. Stein, Packing of $R^{n}$ by certain error spheres, IEEE Trans. Inform. Th. 30 (1984), 356-363.

[25] S. K. Stein and S. Szabó, Algebra and Tiling : Homomorphisms in the Service of Geometry, Carus Mathematical Monographs, \#25, MAA, 1994, 209 pp.

[26] S. Szabó, On mosaics consisting of mutidimensional crosses, Acta Math. Acad. Sci. Hung. 38 (1981), 191-203.

[27] W. Ulrich, Non-binary error correcting codes, The Bell System Technical Journal 36 (1957), 1341-1387. 